\title{
Rapid Near-Optimal VQ Design with a Deterministic Data Net
}

\author{
Michelle Effros ${ }^{1}$ and Leonard J. Schulman ${ }^{2}$ \\ California Institute of Technology · Pasadena, California 91125
}

We present a new algorithm for fixed-rate vector quantizer (VQ) design. A fixed-rate VQ of dimension $d$ and rate $(\log K) / d$ represents each vector in $\mathbb{R}^{d}$ by one of $K$ possible codewords. Given a pdf $p(\mathbf{x})$ on $\mathbb{R}^{d}$ and an integer $K \geq 1$, an optimal VQ $\left\{\mu_{1}^{\star}, \ldots, \mu_{K}^{\star}\right\} \subset \mathbb{R}^{d}$ achieves expected distortion $\Delta=$

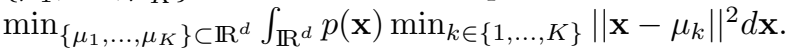

Let $p(\mathbf{x})$ denote the empirical distribution of an $n$ point training set. Optimal VQ design is NP-hard even for $K=2$ [1]. Iterative design finds a locally optimal VQ [4]. For any $\varepsilon>0$, an $\varepsilon$-approximation algorithm guarantees a VQ with expected distortion $D \leq(1+\varepsilon) \Delta$. The best prior $\varepsilon$-approximation algorithms are a deterministic $O\left(\varepsilon^{-2 K^{2} d} n \log ^{K} n\right)$-time $\varepsilon$-approximation [5] and a randomized $O\left(\exp \left(\varepsilon^{-8} K^{3}(\ln K)\left(\ln \frac{1}{\varepsilon}+\ln K\right)\right) n \log ^{K} n\right)$ time $\varepsilon$-approximation [2]. (The problem is called " $K$ clustering" and " $K$-median" in those papers.)

We present a new deterministic $\varepsilon$-approximation algorithm running in time quasilinear in $n$. The algorithm, which also serves as an approximation algorithm for the $d$-dimensional fixed-rate operational distortion-rate function, extends to a variety of network VQ problems.

Define a data net to be a set $\mathcal{Z} \subset \mathbb{R}^{d}$, regions $\left\{A_{z}\right\}_{z \in \mathcal{Z}}$ and mapping $\zeta: \mathbb{R}^{d} \rightarrow \mathcal{Z}$ such that

1. $\int_{A_{z}} p(x)\|x-z\|^{2} d \mathbf{x} \leq \varepsilon \Delta / K$ for all $z \in \mathcal{Z}$

2. For any $\mu \in R^{d}$ and $x \notin A_{\zeta(\mu)}$,

$$
\|x-\zeta(\mu)\|^{2} \leq(1+\varepsilon)\|x-\mu\|^{2} \text {. }
$$

Our deterministic algorithm designs a data net of size $M=c^{d} K^{2}\left(K^{2}+\varepsilon^{-2}\right) \varepsilon^{-d-1}$ for some constant $c$, within time $M n \log \log n$ and then returns the best codebook $\left\{\mu_{1}, \ldots, \mu_{K}\right\} \subset \mathcal{Z}$.

Theorem 1 The above deterministic algorithm finds a rate- $(\log K) / d$ fixed-rate $V Q$ with distortion $D \leq(1+\varepsilon) \Delta$ within time

$$
M n \log \log n+M^{K+1} n .
$$

Subsequent to codebook design, individual encodings can be peformed in time $\log (K / \varepsilon)$ using [3].

We can improve Theorem 1's dependence on $K$ at the expense of $d$. The algorithm can also perform efficient VQ design for simply characterized continuous distributions.

The algorithm generalizes to give $\varepsilon$-approximation algorithms for many network VQ design problems. A few

\footnotetext{
${ }^{1}$ M. Effros (effros@caltech.edu) is partially supported by NSF CCR-0220039 and Caltech's Lee Center for Advanced Networking.

${ }^{2}$ L. J. Schulman (schulman@caltech.edu) is partially supported by NSF CCR-0049092, the Charles Lee Powell Foundation, and a visiting membership at MSRI.
}

examples follow. Many more examples (including all combintations of listed examples) follow similarly.

Multiresolution VQ (MRVQ): A transmitter describes source $X$ to $L$ receivers. Receiver $\ell$ gets only the first $\sum_{i=1}^{\ell} \log K_{i}$ bits of the binary description.

Multiple description VQ (MDVQ): A transmitter sends $L$ packets describing source $X$. Any subset of packets may be lost in transmission.

Side information VQ (SIVQ): A transmitter describes source $X$ to a receiver that has access to side information unavailable to the encoder.

Broadcast VQ (BCVQ): A transmitter describes multiple sources to a family of decoders. Each source is intended for a distinct subset of the receivers, and each component of the description is received by a distinct subset of the receivers.

Joint source-channel VQ (JSCVQ): A transmitter describes a single source to a single receiver. The description may be corrupted during transmission.

Remote source VQ (RSVQ): An encoder observes a noisy copy of the true source and describes it to the decoder. The decoder reconstructs the true source as accurately as possible.

In each example, let $S$ be the number of sources $(S=1$ in all but the BCVQ), $K$ be the maximal number of codewords that can be distinguished by any single decoder, $T$ be the total number of $d$-dimensional codewords in the code, and $\Delta$ be the optimal performance (an expected distortion over some distribution on the reconstructions at different receivers).

Theorems 2-7 In each scenario above, designing a data net of size $M=c^{d} K^{2}\left(K^{2}+\varepsilon^{-2}\right) \varepsilon^{-d-1}$ for each source, and using the best $T$ codewords from those data nets gives a code with performance $D \leq(1+\varepsilon) \Delta$ in time

$$
S M n \log \log n+(S M)^{T+1} n .
$$

\section{REFERENCES}

[1] P. Drineas, A. Frieze, R. Kannan, S. Vempala, and V. Vinay. Clustering in large graphs and matrices. In Proc. 10th Annual ACM-SIAM Symposium on Discrete Algorithms (SODA), 1999.

[2] W. Fernandez de la Vega, M. Karpinski, C. Kenyon, and Y. Rabani. Approximation schemes for clustering problems. In Proc. 35'th Ann. Symp. on Theory of Computing (STOC), 2003.

[3] S. Har-Peled. A replacement for Voronoi diagrams in near linear size. In Proc. 42nd Annual IEEE Symposium on Foundations of Computer Science (FOCS), 2001.

[4] Y. Linde, A. Buzo, and R. M. Gray. An algorithm for vector quantizer design. IEEE Trans. Comm., 28(1):84-95, 1980.

[5] J. Matousek. On approximate geometric $k$-clustering. Discrete 8 Computational Geometry, 24:61-84, 2000. 